This item was submitted to Loughborough's Research Repository by the author.

Items in Figshare are protected by copyright, with all rights reserved, unless otherwise indicated.

\title{
Theoretical and experimental studies of off-the-shelf v-dot probes
}

PLEASE CITE THE PUBLISHED VERSION

https://doi.org/10.1109/TPS.2018.2854971

PUBLISHER

(c) IEEE

VERSION

AM (Accepted Manuscript)

LICENCE

CC BY-NC-ND 4.0

REPOSITORY RECORD

Novac, Bucur M., Renzhen Xiao, Tom Huiskamp, Laurent Pecastaing, Meng Wang, Peter Senior, Antoine Silvestre De Ferron, AJM Pemen, and Mark Rivaletto. 2019. "Theoretical and Experimental Studies of Off-theshelf V-dot Probes”. figshare. https://hdl.handle.net/2134/34321. 


\title{
Theoretical and Experimental Studies of Off-the-Shelf V-dot Probes
}

\author{
Bucur M. Novac, Senior Member, IEEE, Renzhen Xiao, Tom Huiskamp, Laurent Pécastaing, Senior \\ Member, IEEE, Meng Wang, Peter Senior, Antoine S. de Ferron, A.J.M. Pemen, Member, IEEE and \\ Marc Rivaletto
}

\begin{abstract}
The paper introduces the work undertaken to reliably use off-the-shelf differentiating voltage probes attached to coaxial transmission lines. The results obtained prove that indeed such probes are a valid and simple instrument for measuring nanosecond and subnanosecond voltage impulses. As a bonus, the research also highlighted an important challenging phenomenon that appears whenever an attempt is made to measure fast voltage impulses with a differentiating probe positioned too close to the closing switch of a pulse forming line generator.
\end{abstract}

Index Terms-Pulsed power systems, Voltage probes, Pulse Forming Lines

\section{INTRODUCTION}

$\mathrm{T}$ HE measurement of fast transient high-voltages impulses in pulsed power experiments is well-known to be difficult and specialist conferences have been dedicated to it [1]. After being introduced in open literature in 1980 [2], V-dot probes have been successfully used in the next decades to measure fast transient $\mathrm{kV}$ and $\mathrm{MV}$ voltage signals generated by various impulse generators. In studying the published literature, with a few representative examples being [2-8], the general impression is that the design and implementation of a V-dot probe (sometimes termed D-dot or antenna) is something complicated. This feeling arises from the sometimes ingenuous but certainly quite different probe geometries used by various pulsed power laboratories worldwide to solve their specific diagnostic requirements. This paper is however concentrating on the measurement of fast nanosecond and subnanosecond voltage impulses in coaxial transmission lines

Manuscript received in January 2018

Bucur M. Novac and Peter Senior are with the Wolfson School of Mechanical, Electrical and Manufacturing Engineering, Loughborough University, Loughborough, Leicestershire LE11 3TU, UK (e-mail: b.m.novac@lboro.ac.uk).

Renzhen Xiao is with the Northwest Institute of Nuclear Technology, Xi'an 710024, P.R. China (email: xiaorenzhen@tsinghua.org.cn)

Tom Huiskamp and A. J. M. Pemen are with the Electrical Energy Systems Group, Eindhoven University of Technology, 5600 MB Eindhoven, The Netherlands (e-mail: t.huiskamp@tue.nl, a.j.m.pemen@tue.nl).

Laurent Pécastaing, Antoine S. de Feron and Marc Rivaletto are with the Laboratoire SIAME, Equipe Génie Electrique, Université de Pau et des Pays de l'Adour, Hélioparc Pau-Pyrénées, 2 av Angot 64053 Pau Cedex 9, France (e-mail: laurent.pecastaing@univ-pau.fr ).

Meng Wang is with the Institute of Electrical Engineering, Chinese Academy of Sciences, Beijing 100190, P.R. China (email: mengwang@mail.iee.ac.cn).
(TLs) and will demonstrate that use of 'standard' V-dot probes, simply made from off-the-shelf SMA- or N-type connectors and adaptors [9-11], represents a practical solution which potentially offers a simpler alternative to most of the design challenges associated with V-dot probes. The results of the present work are of particular interest for anyone in the domain aiming to obtain results straightforwardly, without losing precious time in developing a new $\mathrm{V}$-dot probe design.

The paper also shows that, when $\mathrm{V}$-dot probes are mounted onto a coaxial pulse forming line (PFL) generator the position must be carefully chosen. This is a recommendation valid for any probe design and not necessarily restricted to those probes made from off-the-shelf items.

\section{STUDIES OF OFF-THE-SHELF N- AND SMA-TYPE V-DOT PROBES MOUNTED ONTO COAXIAL TRANSMISSION LINES}

Numerical 3D electromagnetic analyses have been performed with CST Microwave Studio [12] for N-type female-female (Jack-to-Jack) adapters and for SMA-type panel connectors (Jack, flange mount 'candlesticks'), both used as off-the-shelf V-dot probes in coaxial TLs.

In Fig. 1 the N-type probe, shown mounted on a large outer diameter $(110 \mathrm{~mm})$ TL filled with polyethylene, has been investigated using a $6 \mathrm{GHz}$ Gaussian voltage impulse injected through a CST port along the TL. Its integrated output signal is compared in Fig. 2 with the voltage signal provided by a CST voltage probe placed nearby (V in Fig. 2), with the two traces being indistinguishable! Additionally, (real) experiments were performed with the $\mathrm{N}$-type probes either mounted on a bi-conical adaptor or attached to a high-voltage microwave cable using a metallic supporting element (Fig. 3). In both cases and using a vector network analyzer (VNA), it was proven that up to about $6 \mathrm{GHz}$ their recorded characteristics were practically identical to the CST software predictions (Fig. 4).

Further CST-based studies also showed that by altering the $\mathrm{N}$-type adaptor, either by cutting and removing a slice to allow placing the central pin in direct contact with the TL insulator or by filling the (initially hollow) pin with solder, are not improving the probe characteristics. The best remains to leave the off-the-shelf item as made by the manufacturer.

When used in a PFL generator, SMA-type V-dot probes were mounted onto a HV coaxial microwave cable type 


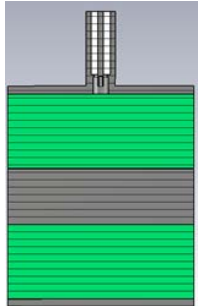

a)

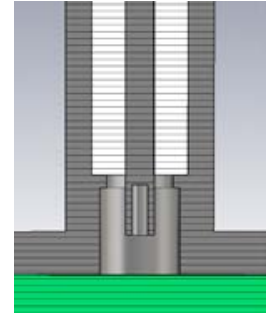

b)

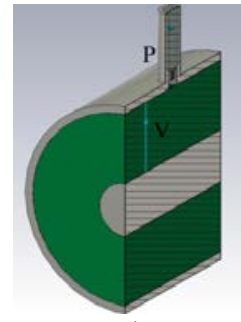

c)
Fig. 1. a) CST model of an N-type adaptor used as a V-dot probe mounted onto a coaxial transmission line. b) Details of the probe. c) A CST voltage probe $(\mathrm{V})$ is used to check the quality of signals generated by the differential V-dot probe (P).

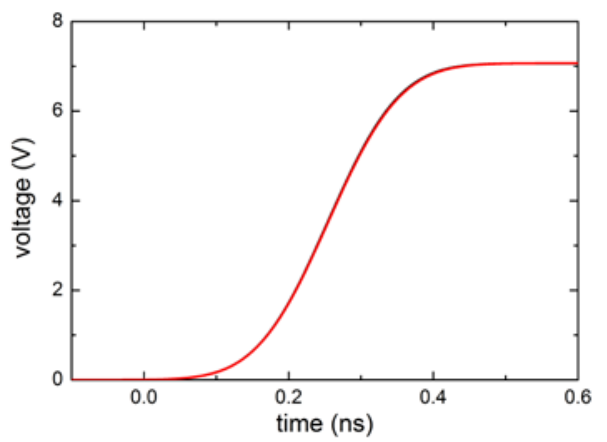

Fig. 2. The integrated output from an N-type V-dot probe (P in Fig. 1) is compared with the signal from a CST voltage probe (V in Fig. 1). The two signals are identical when a $6 \mathrm{GHz}$ Gaussian signal is injected through a CST port along the transmission line.

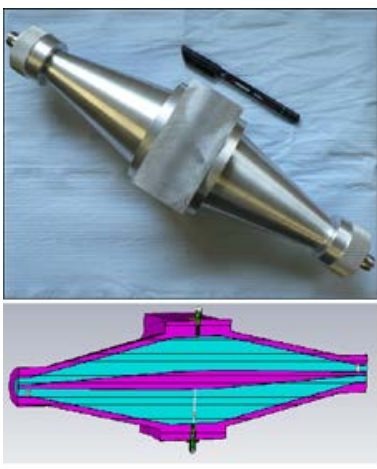

a)

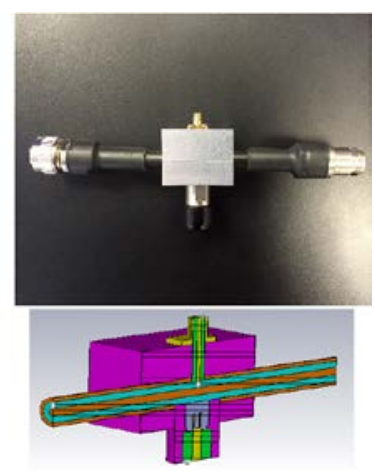

b)
Fig. 3. Experiments with the N-type probe attached to:

a) Bi-conical adaptor; b) High-voltage microwave cable using a metallic supporting element. For both experiments the corresponding CST model is shown below.

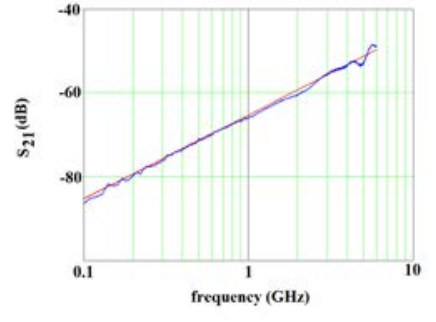

a)

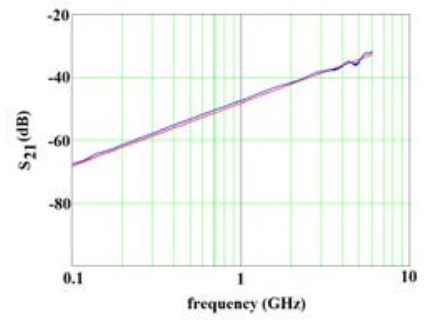

b)
Fig. 4. The variation with frequency over a large bandwidth of the N-type probe $S_{21}$ parameter obtained from experiments using a VNA is successfully compared with the corresponding CST predictions. a) and b) are in reference to Fig. 3

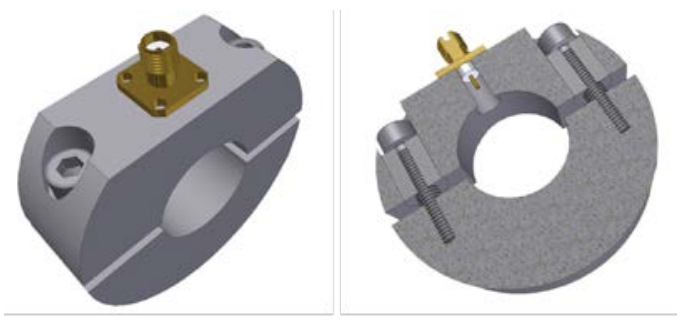

(a) (b)

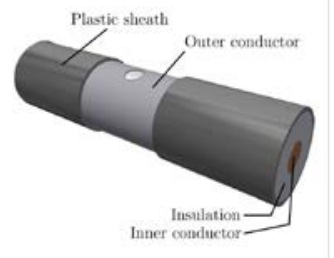

(c)

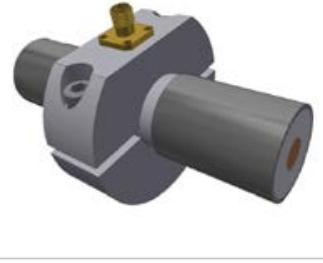

Fig. 5. SMA-type V-dot probe mounted onto a HV microwave cable (a) Drawing of the SMA-based V-dot probe. b) Cut-away view: the tip of the bulkhead connector is the probe's electrode (c) HV microwave SA24272 cable with a hole in the outer conductor (d) V-dot sensor mounted onto cable. The drawings are taken from [9].

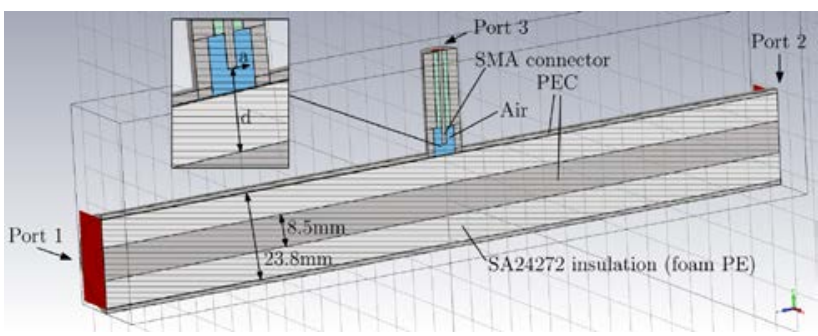

Fig. 6. CST model for the SMA-type V-dot probe shown in Fig. 5. Ports 1, 2 and 3 correspond to the input of the cable, the output of the cable and the output of the V-dot probe, respectively. Distances 'a' and 'd' are used in defining the mounting precision

SA24272 having a diameter of $23.8 \mathrm{~mm}$ (Fig. 5) [9]. CSTbased numerical analysis (Fig. 6) and real experiments using a VNA proved that the experimental data matches the CST predictions over a large bandwidth: between $100 \mathrm{MHz}$ up to well inside the $\mathrm{GHz}$ domain. As shown in Fig. 7, the difference between experimental data and theoretical predictions was found to be on average around $0.3 \mathrm{~dB}$, which represents an error in measured amplitudes of about 3.5\%.

\section{CALIBRATION ISSUES}

Can off-the-shelf probes attached to coaxial TLs be calibrated using only CST modelling? The answer to this question could be either negative or positive, as it depends on the mechanical mounting precision!

For example, in the case of small size transmission lines, the answer is rather negative. In such a case it is always a good practice to validate the initial CST calibration using a VNA. It is not a surprise that, to rely solely on CST calibration, the mounting of probes on a small-size transmission line is demanding. For example, for the SMAtype probe mounted on a $23.8 \mathrm{~mm}$ diameter transmission line, a $\pm 200 \mu \mathrm{m}$ radial positioning error is predicted by CST to change the voltage amplitude by $15.5 \%$ and $19.4 \%$, respectively! The results of a detailed CST study on the 
MS\#TPS11082

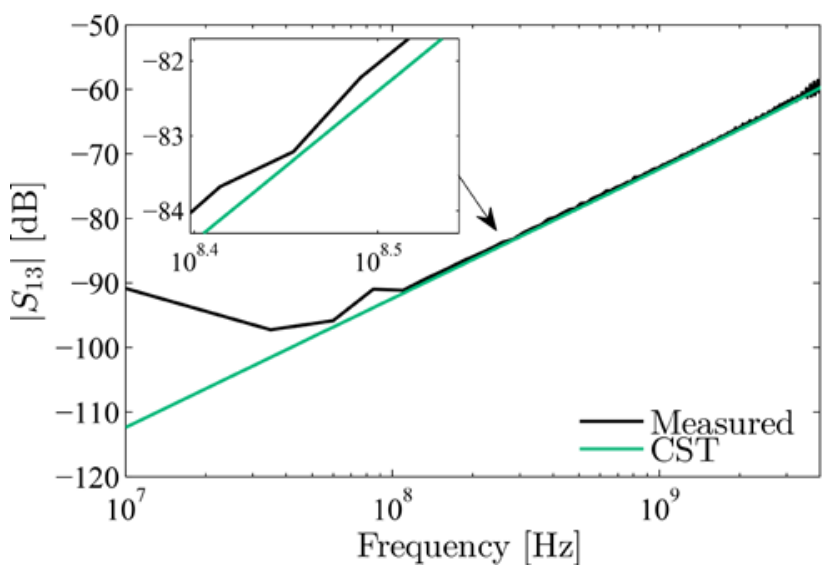

Fig. 7. Comparison between VNA measured and CST simulated $\mathrm{S}_{13^{-}}$ parameters for the SMA-type V-dot sensor presented in Fig. 5.

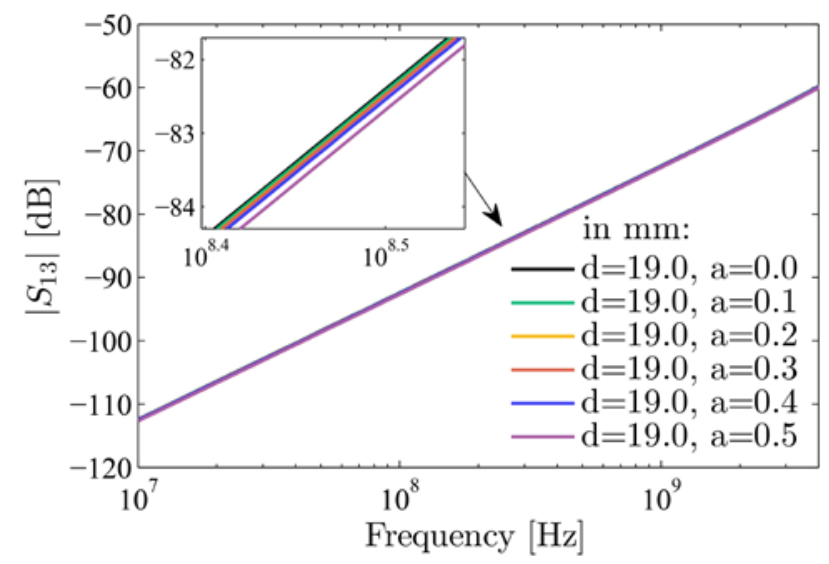

i)

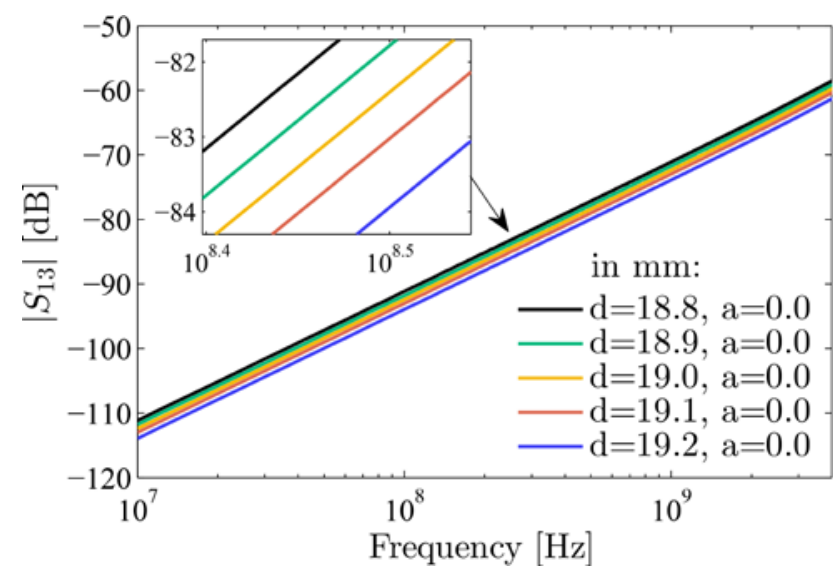

ii)

Fig. 8. Studies of the influence on the S-parameter of various characteristics related to mounting SMA-connectors on a HV cable i) influence of the offset 'a' from its center position ii) influence of distance 'd' to HV electrode. The distances 'a' and 'd' are as in Fig. 6.

influence of mounting precision on the S-parameter are presented in Fig. 8. The influence of the distance from the tip of the SMA connector to the high voltage conductor (indicated by 'd' in Fig. 6) and the shift of the SMA connector axis from the axis of the hole in the shield of the cable (indicated by ' $a$ ' in Fig. 6) are both considered.

However, for large diameter TLs the answer is rather positive. In such a case, the technique using a VNA is in practice expensive (both in time and money), because high bandwidth bi-conical adaptors require careful design and manufacturing. On the other hand, for a large diameter TL the use of CST results to calibrate the V-dot probe is realistically possible, as the required mounting precision is less demanding. For example, for N-type probes mounted on a $110 \mathrm{~mm}$ diameter TL, for the same $\pm 200 \mu \mathrm{m}$ radial positioning error as above, the voltage amplitude is predicted by CST to only change between $1 \%$ and $5 \%$, respectively!

A final note about calibration concerns the signal cables used to connect the V-dot sensors to the measuring instrument (e.g., the oscilloscope). When measuring high-frequency signals (i.e., in the $\mathrm{GHz}$ range), such cables can introduce a supplementary attenuation to the measured signals [9, 10]. It is important to always use as short as possible and extremely low-loss cables for such measurements. Alternatively, the measuring system can be calibrated for the loss of these cables (especially if they are very long), either by de-embedding their response in the measuring instrument, or by numerical reconstruction $[9,10,13]$.

\section{A GENERAL ISSUE RELATED TO USING ANY TYPE OF V-DOT PROBES IN PFLS}

Fig. 9 shows results obtained with an N-type V-dot probe mounted in a coaxial TL, at the output of a bipolar former [14] and about $100 \mathrm{~mm}$ from its spark-gap. The TL has an open end and the measured reflected signals seem to look much 'better' than the direct signals i.e., they are less distorted. As seen in Fig. 9, the distortions present in the direct signal are slightly changing from shot to shot. Fig. 10 shows similar results obtained with an improved version of the bipolar former. This time the V-dot probe is mounted further from the bipolar former spark-gap i.e., at a distance of about $350 \mathrm{~mm}$. As a consequence, both the direct and the reflected signals have practically the same shape! The results above strongly suggest that placing a V-dot probe too close to a spark-gap may generate distorted signals. To understand this phenomenon better, a detailed CST study was undertaken using the arrangement presented in Fig. 11, with two probes (P) mounted onto a PFL and their signals compared with CST voltage probes (V). Firstly, in order to investigate if the phenomenon can be avoided using a better designed probe, three different V-dot probes were considered, as presented in Fig. 12: N-type, 'normal' and 'shielded'. The CST analysis demonstrated there is very little difference between the signals generated by these probes, as they are all affected when mounted too close to a spark-gap! It was also found that the phenomenon is enhanced if the PFL diameter is larger, but mostly if the spark-gap breakdown plasma channel is not situated along the central axis (see Fig. 13). As in a spark-gap the plasma channel position changes from shot to shot (Fig. 13), this may explain the fact that the amount of signal distortion also slightly differs from shot to shot (Fig. 14).

A further study showed the phenomenon being related to the spark-gap closure which generates non-TEM mode 
attenuating or propagating (depending on the size of the TL) electromagnetic waves (besides a TEM mode wave) in the vicinity of the spark gap. These non-TEM mode waves may have electric-field components that are not purely perpendicular to the axis of the coaxial line (Fig. 15). As a consequence, the very fast time-of-change of the non-radial electric field components (Fig. 15a and 15b) do not allow an antenna to behave like a V-dot probe. For comparison, for a V-dot probe placed in a TL (i.e., no spark-gap present) the non-radial components play only a minor role (Fig. 15c). For a more detailed explanation let us consider the equivalent electric circuit of a V-dot probe [2], where the essential role is played by the capacitance between the pin (antenna) and the HV central electrode of the PFL. This capacitance is related to the radial electric field component generated inside the line. If the time rate-of-change of the other electric field components begins to compete with the time-rate of change of the radial component, the antenna will not behave like a V-dot probe i.e., its output will not be proportional to the time-rate-of-change of the voltage impulse travelling along the line. However, as the distance from the spark-gap at which the probe is mounted increases, this unwanted effect decreases because most of the non-TEM modes are attenuating modes. A study of the propagation of high-order modes in a transmission line is presented in Appendix 1.

The optimum position to mount a V-dot probe depends on the PFL diameter, with a small diameter being less demanding because a smaller diameter coaxial structure supports less higher-order propagating wave modes and the higher-order attenuating wave modes will attenuate over a shorter distance [15-17].

However, a simple general rule would be to place the probe as far from a spark-gap as allowed by the experimental arrangement.

\section{CONCLUSIONS}

The paper demonstrates, both theoretically and practically, that V-dot probes made from off-the-shelf $\mathrm{N}$-type adaptors or SMA type connectors can be successfully used to measure fast transient voltage impulses in coaxial TLs. We are not advocating replacing the presently used V-dot probes with offthe-shelf components. We are only advising newcomers not to be intimidated by sophisticated literature designs and be encouraged to straightforwardly install and use off-the-shelf V-dot probes. As a bonus, the difficult task of calibrating such probes when used in large diameter transmission lines can in most cases rely solely on their CST modelling.

The paper also highlights an important perturbing factor inside coaxial PFLs that requires mounting any type of V-dot probes as far as possible from the PFL spark-gap, because of the unwanted influences of higher-order non-TEM wave modes.

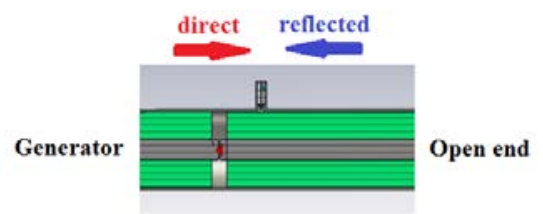

a)
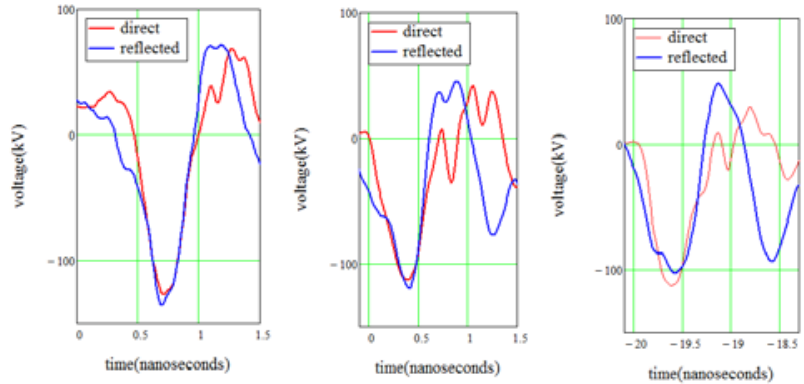

b)

Fig. 9. a) N-type V-dot probe shown mounted onto an open ended coaxial transmission line at the output of a bipolar former about $100 \mathrm{~mm}$ from its spark-gap. b) Integrated voltage probe signals obtained from three different shots. For convenience, the reflected signal is shown as being generated at the same time as the direct signal.
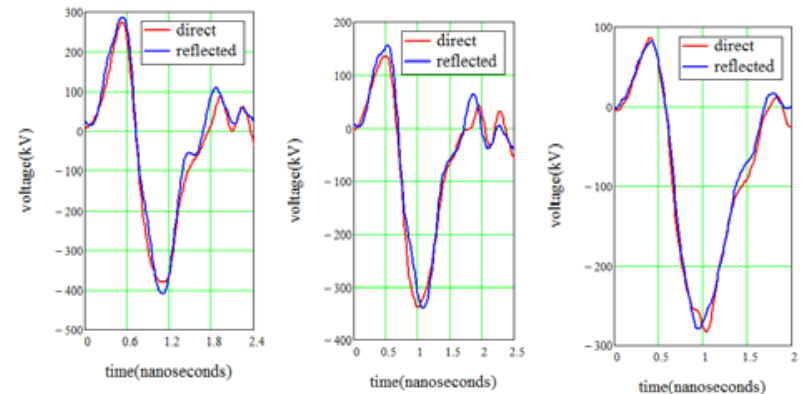

Fig. 10. Same type of results as in Fig.8, with the probe mounted about $350 \mathrm{~mm}$ from the spark-gap.

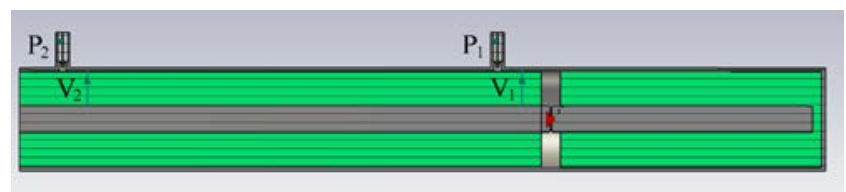

Fig. 11. CST model of a PFL with two N-type V-dot probes installed $\left(\mathrm{P}_{1}\right.$ and $\left.\mathrm{P}_{2}\right)$. For each probe there is a CST voltage sensor $\left(\mathrm{V}_{1}\right.$ and $\left.\mathrm{V}_{2}\right)$.

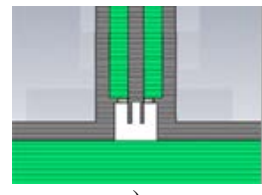

a)

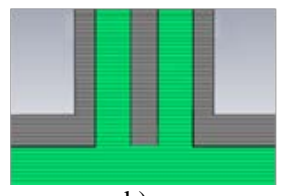

b)

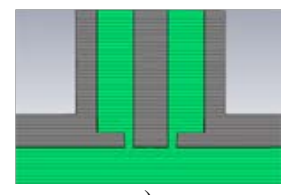

c)
Fig. 12. Three possible V-dot designs: a) based on an N-type adaptor b) 'normal', with the central electrode (antenna) in contact with the transmission line dielectric and c) 'shielded' 
MS\#TPS11082

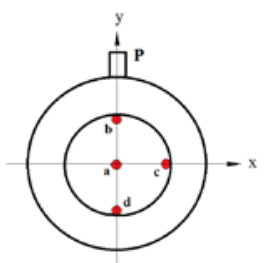

Fig. 13. Cross-section of a coaxial PFL showing four possible loci (a, b, c, d) for the spark-gap plasma channel in respect to the position of the V-dot probe P.
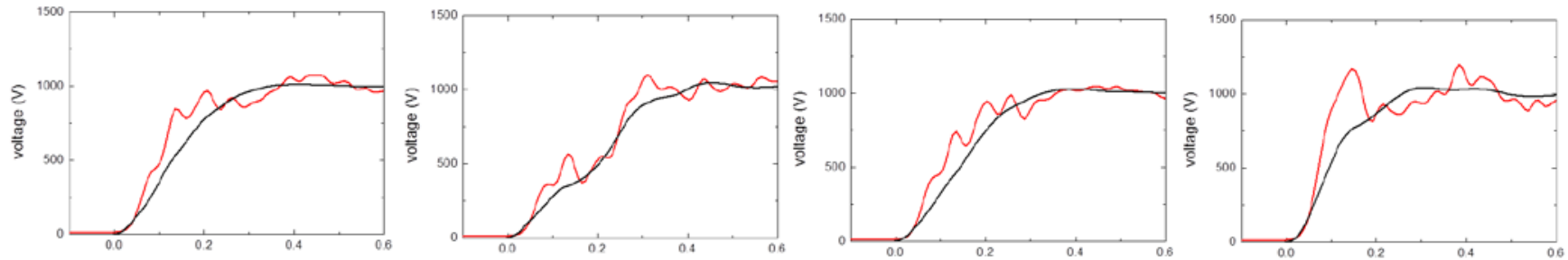

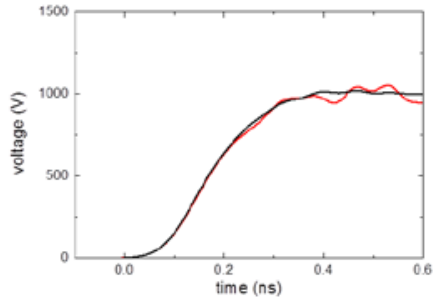

a)

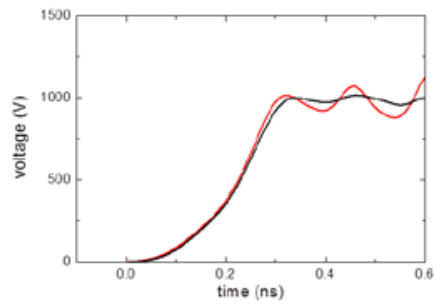

b)

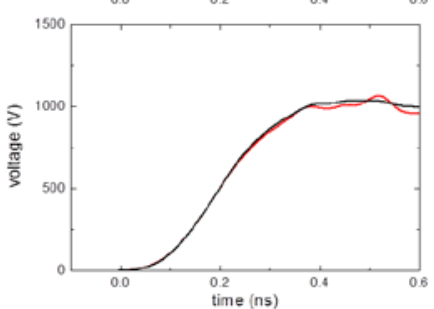

c)

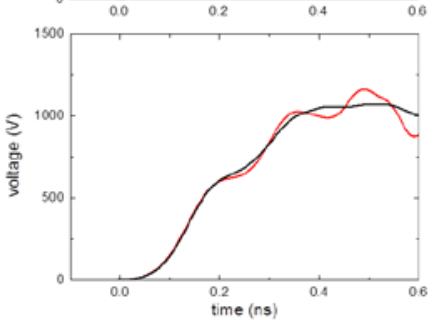

d)

Fig. 14. The influence of the plasma channel position in a spark gap on the signal distortion of the two V-dot probes of Fig. 10. Upper row: integrated signals from the probe $\mathrm{P}_{1}$ compared with the corresponding CST voltage probe $\mathrm{V}_{1}$. Lower row: same for the pair $\mathrm{P}_{2}$ and $\mathrm{V}_{2}$. Columns a, b, c and d correspond to the positions of the spark-gap plasma channel, as in Fig. 13.
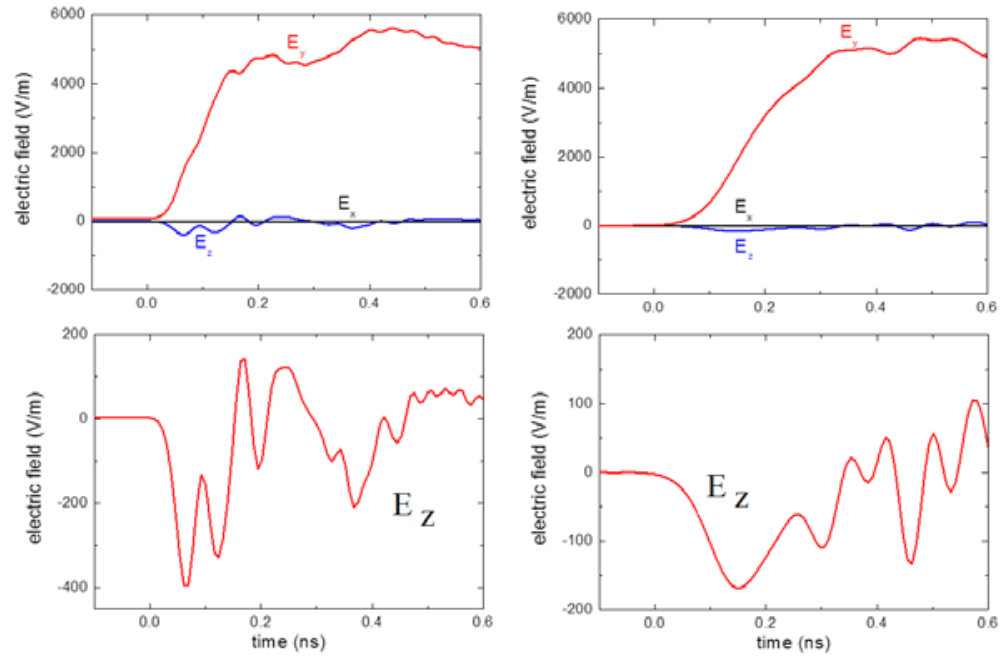

a)

b)

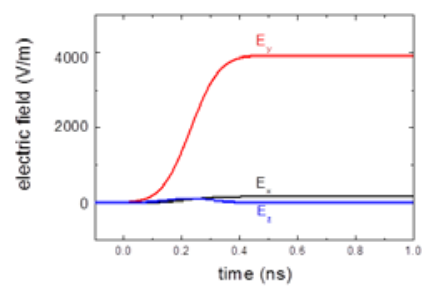

c)

Fig. 15. Time dependence of the electric field vector components in a PFL a) for probe $\mathrm{P}_{1}$ and b) for probe $\mathrm{P}_{2}$. The probes are positioned as in Fig.11. c) the time dependence of the electric field vector components for a V-dot probe placed in a TL as in Fig. 1. Electric field Ox and Oy axes are as in Fig.13, with the Oz along the transmission line axis. 


\section{APPENDIX 1}

As suggested by CST simulations, high-order modes are probably excited due to the random breakdown of spark-gap switch, forming off-axis (asymmetric) plasma channels. In addition, breakdown processes leading up to and during the early stages of the spark-gap switching can have such a highfrequency nature, that higher-order modes can be excited (even though most will be attenuating modes).

In what follows, a brief analysis of the TM and TE propagating modes in a coaxial waveguide is provided.

The field-component expressions in cylindrical coordinates for the TM modes are [1]:

$$
\begin{aligned}
& E_{r}=-j \beta T U_{0}\left[N_{n}(T a) J_{n}^{\prime}(T r)-J_{n}(T a) N_{n}^{\prime}(T r)\right] \cos (n \phi) e^{-j \beta z}, \\
& E_{\phi}=-j \frac{\beta n}{r} U_{0}\left[N_{n}(T a) J_{n}(T r)-J_{n}(T a) N_{n}(T r)\right] \sin (n \phi) e^{-j \beta z}, \\
& E_{z}=T^{2} U_{0}\left[N_{n}(T a) J_{n}(T r)-J_{n}(T a) N_{n}(T r)\right] \cos (n \phi) e^{-j \beta z}, \\
& H_{r}=-j \frac{\omega \varepsilon n}{r} U_{0}\left[N_{n}(T a) J_{n}(T r)-J_{n}(T a) N_{n}(T r)\right] \sin (n \phi) e^{-j \beta z}, \\
& H_{\phi}=-j \omega \varepsilon T U_{0}\left[N_{n}(T a) J_{n}^{\prime}(T r)-J_{n}(T a) N_{n}{ }^{\prime}(T r)\right] \cos (n \phi) e^{-j \beta z}, \\
& H_{z}=0 .
\end{aligned}
$$

where $J_{n}$ and $N_{n}$ are the $n$-th order first kind and second kind Bessel functions and $J_{n}{ }^{\prime}$ and $N_{n}{ }^{\prime}$ are their corresponding differentials, respectively; $U_{0}$ is the normalized field amplitude for the TM modes; $T$ is the transverse wavenumber and $\beta$ is the longitudinal wave-number satisfying the relation $k^{2}=T^{2}+\beta^{2}$, where $k=2 \pi f \sqrt{\mu_{r} \varepsilon_{r}} / c$.

The dispersion equation for the TM mode in a coaxial waveguide is:

$$
J_{n}(T b) N_{n}(T a)-J_{n}(T a) N_{n}(T b)=0 .
$$

where $a$ and $b$ are the outer and inner radii of the coaxial waveguide, respectively.

The field-component expressions (in cylindrical coordinates) for the TE modes are [1]:

$$
\begin{aligned}
& E_{r}=\frac{j \omega \mu n}{r} V_{0}\left[N_{n}{ }^{\prime}(T a) J_{n}(T r)-J_{n}{ }^{\prime}(T a) N_{n}(T r)\right] \sin (n \phi) e^{-j \beta z}, \\
& E_{\phi}=-j \omega \mu T V_{0}\left[N_{n}^{\prime}(T a) J_{n}{ }^{\prime}(T r)-J_{n}{ }^{\prime}(T a) N_{n}^{\prime}(T r)\right] \cos (n \phi) e^{-j \beta z}, \\
& E_{z}=0, \\
& H_{r}=-j \beta T V_{0}\left[N_{n}^{\prime}(T a) J_{n}{ }^{\prime}(T r)-J_{n}{ }^{\prime}(T a) N_{n}{ }^{\prime}(T r)\right] \cos (n \phi) e^{-j \beta z}, \\
& H_{\phi}=\frac{j \beta n}{r} V_{0}\left[N_{n}{ }^{\prime}(T a) J_{n}(T r)-J_{n}{ }^{\prime}(T a) N_{n}(T r)\right] \sin (n \phi) e^{-j \beta z}, \\
& H_{z}=T^{2} V_{0}\left[N_{n}{ }^{\prime}(T a) J_{n}(T r)-J_{n}{ }^{\prime}(T a) N_{n}(T r)\right] \cos (n \phi) e^{-j \beta z},
\end{aligned}
$$

where $V_{0}$ is the normalized field amplitude for the TE modes.
The corresponding dispersion equation for the TE mode in a coaxial line is given by:

$J_{n}{ }^{\prime}(T b) N_{n}{ }^{\prime}(T a)-J_{n}{ }^{\prime}(T a) N_{n}{ }^{\prime}(T b)=0$.

$T$ can be obtained from the dispersion equations, but we decided to use CST to obtain the cutoff frequency $f_{c}$, from which $T$ can be obtained from:

$T=\frac{2 \pi f_{c}}{c} \sqrt{\mu_{r} \varepsilon_{r}}$

while the corresponding group velocity $v_{g}$ is given by:

$v_{g}=\frac{c}{\sqrt{\mu_{r} \varepsilon_{r}}} \sqrt{1-\frac{T^{2}}{k^{2}}}$

which can be re-written as:

$v_{g}=\frac{c}{\sqrt{\mu_{r} \varepsilon_{r}}} \sqrt{1-\frac{\lambda^{2}}{\lambda_{c}^{2}}}$

where $\lambda_{c}$ is the cutoff wavelength $\left(\lambda_{c} f_{c}=c / \sqrt{\varepsilon_{r}}\right)$.

As a first example let us consider $a=20 \mathrm{~mm}, b=6 \mathrm{~mm}$, and $\varepsilon_{r}=2.1$ for which the cut-off frequencies of the TE 11 and the $\mathrm{TE}_{21}$ mode are $2.6 \mathrm{GHz}$ and $4.89 \mathrm{GHz}$, respectively. We note that the cut-off frequencies of the $\mathrm{TM}_{01}$ mode and $\mathrm{TM}_{11}$ mode are $7.26 \mathrm{GHz}$ and $7.75 \mathrm{GHz}$ respectively. These frequencies are much higher than those for the TE modes and therefore are more difficult to be excited. As $k^{2}=T^{2}+\beta^{2}$, for an attenuating wave with the frequency less than the cutoff frequency $\beta$ will be an imaginary number and for the present case $\beta=-21.6 i$. Using the field-component Eqs (1) and (3) it is obvious that the term $e^{-j \beta z}$ is attenuated as the wave travels along the coordinate $\mathrm{z}$, or in other words the $\mathrm{TE}_{11}$ mode will have an e-fold decay (1/e ) after about $5 \mathrm{~cm}$.

It is possible that the TEM, $\mathrm{TE}_{11}$ and $\mathrm{TE}_{21}$ mode are all excited at the same time by the spark-gap switch. Let us assume the frequencies of the excited $\mathrm{TE}_{11}$ mode and $\mathrm{TE}_{21}$ mode to be $5 \mathrm{GHz}$, and $10 \mathrm{GHz}$, respectively. In such conditions, it can be calculated that the group velocities of the TEM mode, $\mathrm{TE}_{11}$ mode and $\mathrm{TE}_{21}$ mode are $20.7 \mathrm{~cm} / \mathrm{ns}, 17.68 \mathrm{~cm} / \mathrm{ns}$, and $18.06 \mathrm{~cm} / \mathrm{ns}$, respectively. If the pulse rise time is $t_{r}$, in order to avoid the higher-order mode disturbing the rise edge, the length $\mathrm{L}$ of transmission line between the spark gap and the $\mathrm{V}$ dot probe should satisfy the condition:

$\frac{L}{v_{T E_{21}}}-\frac{L}{v_{T E M}}>t_{r}$

In the present case for $t_{r}=0.2 \mathrm{~ns}, L>28 \mathrm{~cm}$. For the $\mathrm{TM}_{01}$ mode and $\mathrm{TM}_{11}$ mode with frequencies of $10 \mathrm{GHz}$, the group velocities are $14.2 \mathrm{~cm} / \mathrm{ns}$ and $13.1 \mathrm{~cm} / \mathrm{ns}$, respectively and therefore $\mathrm{L}>9 \mathrm{~cm}$. From this point of view, for the same frequency, the mode with lower cutoff frequency will need longer distance to avoid the disturbance, so the higher-order mode with a higher cutoff frequency can be neglected.

As a second example let us consider $a=60 \mathrm{~mm}, b=18 \mathrm{~mm}$, $\varepsilon_{r}=2.1$, for which the cutoff frequencies of the $\mathrm{TE}_{11}$ mode and $\mathrm{TE}_{21}$ mode are $0.868 \mathrm{GHz}$ and $1.63 \mathrm{GHz}$, respectively. For 
the same frequencies of the $\mathrm{TE}_{11}$ mode and $\mathrm{TE}_{21}$ mode as before (i.e., $5 \mathrm{GHz}$ and $10 \mathrm{GHz}$ ), the group velocities of the TEM mode, $\mathrm{TE}_{11}$ mode and $\mathrm{TE}_{21}$ mode are $20.7 \mathrm{~cm} / \mathrm{ns}$, $20.39 \mathrm{~cm} / \mathrm{ns}$, and $20.40 \mathrm{~cm} / \mathrm{ns}$, respectively. In this case for $t_{r}=0.2 \mathrm{~ns}, L>2.8 \mathrm{~m}$. We note that the group velocities are dependent on both the cut-off frequency and the frequency of electromagnetic wave. On the other hand, the cut-off frequency is determined by the dimensions of the coaxial waveguide. Because the $\mathrm{TE}_{11}$ and $\mathrm{TE}_{21}$ mode are the modes with the lowest cutoff frequencies, for other modes with higher cutoff frequency less distance will be required to avoid any disturbance.

As a conclusion, to avoid a higher mode disturbance, the distance a V-dot probe can be placed from a spark gap and measure the correct rise time increases with the radius of the waveguide and with the frequency. Finally, the larger the frequency difference between the cutoff frequency and the high frequency signal, the longer this distance will be.

\section{REFERENCES}

[1] R. H. McKnight and R. E. Hebner Jr., Editors, "Measurement of Electrical Quantities in Pulsed Power Systems" National Bureau of Standards, Boulder, Colorado, 2-4 March 1981

[2] C. A. Ekdahl, "Voltage and current sensors for a high-density z-pinch experiment”, Rev. Sci. Instrum. vol. 51, no. 12, pp. 1645-1648, 1980

[3] S. Burkhart, "Coaxial E-field probe for high-power microwave measurement, IEEE Trans. Microwave Theory and Techniques, vol. MTT-33, no.3, pp. 262-265, 1985

[4] A. Küchler, J. Dams, Th. Dunz and A.J. Schwab, "Combined E- and HField Probe for Traveling Wave Analysis in Pulse Power Generators", Proc. IEEE Int. Pulsed Power Conf., pp. 381-384, 1985.

[5] P. Choi and M. Favre, "A Fast Capacitive Voltage Monitor for Low Impedance Pulse Lines”, Proc. IEEE Int. Pulsed Power Conf., pp. 880885, 1995

[6] K. W. Struve, M. L. Horry and R. B. Spielman, "Design, Calibration, and Performance of Water Diagnostics on the PBFA Z", Proc. IEEE Int. Pulsed Power Conf., pp. 1303-1308, 1997.

[7] P. Choi and M. Favre, "Fast pulsed hollow cathode capillary discharge device”, Rev. Sci. Instr., vol. 69, no. 9, pp. 3118-3122, 1998.

[8] T. C. Wagoner et al., "Differential-output B-dot and D-dot monitors for current and voltage measurements on a 20-MA, 3-MV pulsed-power accelerator“, Phys. Rev. ST Accel. Beams, vol. 11, 1s00401 (2008)

[9] T. Huiskamp, F. J. C. M. Beckers, E. J. M. van Heesch and A. J. M. Pemen, "First Implementation of a Subnanosecond Rise-Time, Variable Pulse Duration, Variable Amplitude, Repetitive, High-Voltage Pulse Source," IEEE Trans. Plasma Sci. vol. 42, pp. 859-867, 2014

[10] T. Huiskamp, F. J. C. M. Beckers, E. J. M. van Heesch and A. J. M. Pemen, "B-dot and D-dot Sensors for (Sub)Nanosecond High-Voltage and High-Current Pulse Measurements," IEEE Sens. J., vol. 16, pp. 3792-3801, 2016.

[11] B. M. Novac, Meng Wang, I. R. Smith and P. Senior, "A 10 GW TeslaDriven Blumlein Pulsed Power Generator”, IEEE Trans. Plasma Sci., vol. 42, No. 10, pp. 2876-2885, 2014.

[12] CST, accessed on January 2018. [Online]. Available https://www.cst.com/Products

[13] W. B. Boyer, Sandia National Laboratories Report, No. SAND87-3072, 1987.

[14] Meng Wang, B. M. Novac, L. Pecastaing and I.R. Smith, "Bipolar Modulation of the Output of a 10-GW Pulsed Power Generator", IEEE Trans. Plasma Sci., vol. 44, No. 10, pp. 1971-1977, 2016.

[15] T. Huiskamp, F.J.C.M. Beckers, E.J.M. van Heesch and A.J.M. Pemen, "First Implementation of a Subnanosecond Rise-Time, Variable Pulse Duration, Variable Amplitude, Repetitive, High-Voltage Pulse Source,” IEEE Trans. Plasma Sci., vol. 42, pp. 859-867, 2014.

[16] S. Ramo, J. Whinnery, and T. Van Duzer, Fields and Waves in Communication Electronics. New York, NY, USA: Wiley, 1993
[17] N. Marcuvitz, Waveguide Handbook, vol. 21. Lucknow, India: Inst. Eng. Technol., 1951.

[18] K. Zhang, D. Li, Electromagnetic Theory for Microwaves and Optoelectronics, Springer 2008. 


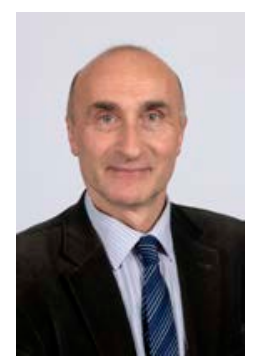

Bucur M. Novac (M’06 - SM’08) received the M.Sc. and Ph.D. degrees in 1977 and 1989, respectively, both from the University of Bucharest. He joined the Loughborough University, UK in 1998 and is currently Professor of Pulsed Power. His research interests include compact and repetitive high-power systems, explosively and electromagnetically driven magnetic flux compression generators and their applications, electromagnetic launchers, ultrafast magneto and electro-optic sensors and 2-D modeling of pulsed-power systems. He has co-authored two books on explosive pulsed power and has published more than 200 refereed papers and conference contributions.

Prof. Novac is a voting member of the Pulsed Power Science \& Technology Committee in the IEEE Nuclear and Plasma Science Society. He is also a member of the International Steering Committees for both the MEGAGAUSS Conferences and for the Euro-Asian Pulsed Power Conferences. He is also member of the organizing committee for the IEEE International Power Modulator and High Voltage Conference and co-chairman of the UK Pulsed Power Symposium. Prof. Novac is a Chartered Engineer and a Fellow of The Institution of Engineering and Technology (IET), UK.

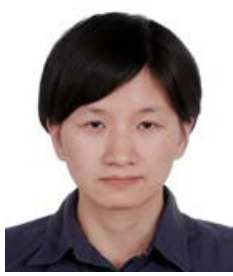

Renzhen Xiao received the Ph.D. degree in nuclear science and technology from Tsinghua University, Beijing, China, in 2007.

Since 2002, she has been with the Northwest Institute of Nuclear Technology, Xi'an, China. Her research interests include relativistic electronics and high power microwave generation and transmission.

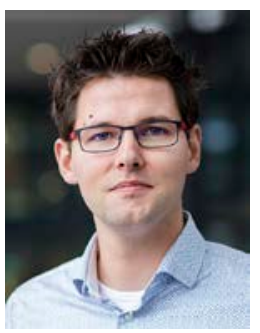

Tom Huiskamp was born in Den Dolder, The Netherlands, in 1985. He received the M.Sc. (Hons.) and Ph.D. (Hons.) degrees in electrical engineering from the Eindhoven University of Technology, Eindhoven, The Netherlands, in 2011 and 2015, respectively. His master's dissertation focused on the application of pulsed power to InnoPhysics' PlasmaPrint technology.

He was a Ph.D. Researcher with the Electrical Energy Systems Group, Eindhoven University of Technology, where he was involved in nanosecond pulsed power-induced corona plasmas and currently works as an Assistant Professor. His current research interests include nanosecond pulse generation, solid-state pulse topologies, and transient plasmas for environmental applications.

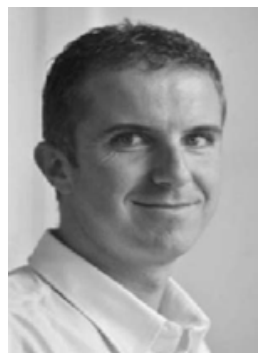

Laurent Pécastaing (M’13 - SM'17) received the Ph.D. degree and the Research Directorship Habilitation in electrical engineering from the Université de Pau et des Pays de l'Adour (UPPA), Pau, France, in 2001 and 2010, respectively. Since 2016, he is a Full Professor with the SIAME Laboratory, UPPA. He is the head of the Electrical Engineering team of the SIAME Laboratory and he is also Director of a Common Laboratory between CEA and UPPA.

His research interests are focused on high-power microwave (HPM) sources, compact pulsed power devices and ultrafast transient probes. Laurent Pécastaing is the Chairman of the next Euro-Asian Pulsed Power Conference (EAPPC) - BEAMS conference to be held in France in 2020.

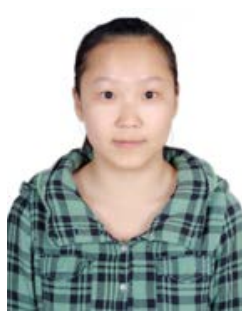

Meng Wang was born in China. She received the B.Sc. degree from Huazhong University of Science and Technology, China, in 2011, the M.Sc. degree from the University College London, UK, in 2012 and the Ph.D degree from Loughborough University, UK, in 2016. She is currently Ph.D. researcher in Institute of Electrical Engineering, Chinese Academy of Sciences. Her current research interests include repetitive high voltage nanosecond pulsed power generator, magnetic switches and pulse forming topologies.

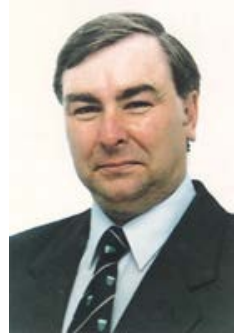

Peter Senior (M'16) received an Honours Degree in Physics with Electronics from Leicester University. He is a member of the Institute of Physics and a Chartered Physicist. He began his career in the Electronic and Electrical Engineering Department at Loughborough University researching Ultrasonic Nondestructive Evaluation, extending into Nonlinear (Finite Amplitude) Acoustics. He was one of the founder members of the Pulsed Power Research Group. He has worked on Propellant and Explosive Pulsed MHD Generators, and also produced systems to support a Flux Compressor Programme. Other work has included High Efficiency Launchers and the Electromagnetic Protection of Armoured Vehicles. He has produced a number of Transportable High Energy, High Power Systems, varying in size between fitting into a van, and up to two ISO Containers, supplied by diesel generators. This has required expertise in the Health and Safety Aspects of integrating Pulsed Power systems with the equipment of sponsoring and collaborating bodies.

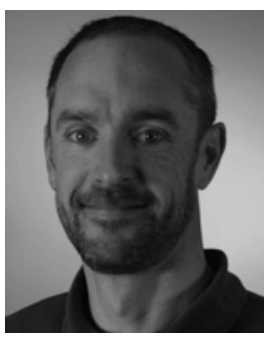

Antoine Silvestre de Ferron received the Ph.D. degree in electrical engineering from the Université de Pau et des Pays de l’Adour (UPPA), Pau, France, in 2006. From 2006 to 2008, he was a Researcher with the Atomic Energy Comission (CEA), Le Barp, France-a French-government-funded technological research organization. He is currently an Engineer with the Laboratoire SIAME, UPPA.

His current research interests include high pulsed power generation for military and civil applications and combined high-voltage transient probes.

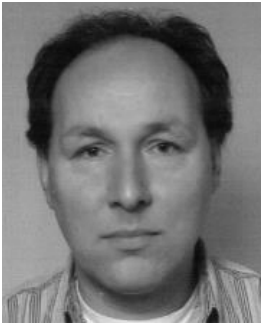

A. J. M. Pemen received the Ph.D. degree in electrical engineering from the Eindhoven University of Technology, Eindhoven, The Netherlands, in 2000.

He is currently a Professor and a Chair of the Electrical Energy Systems Group, Eindhoven University of Technology, where he leads research on smart grids, power grid components, power quality, and EMC and pulsed power technology.

His current research interests include pulsed power technology focuses on advanced, repetitive, nanosecond pulsed power methods for the generation, characterization and utilization of atmospheric pressure, and transient plasmas.

Marc Rivaletto received the bachelor's degree from the Supelec Electrical

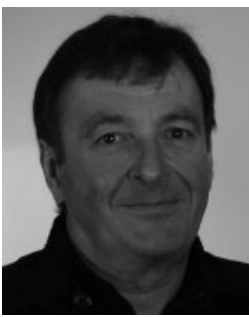
Engineering School, Gif-sur-Yvette, France, in 1984, and the Ph.D. degree in electrical engineering from Pau University, Pau, France, in 1997. He is currently a Lecturer with Pau University and with the SIAME Laboratory, Université de Pau et des Pays de l'Adour, Pau.

His current research interests include high-power microwave sources, and compact pulsed power devices including pulse forming lines, compact Marx generators, or resonant transformers. 